\title{
COMPLETE TRANSFORMER MODEL FOR ELECTROMAGNETIC TRANSIENTS
}

\author{
Francisco de León ${ }^{*} \quad$ Adam Semlyen \\ Department of Electrical Engineering \\ University of Toronto \\ Toronto, Ontario, Canada, M5S 1A4 \\ * Currently with Instituto Politécnico Nacional, Mexico
}

Abstract- A complete, three phase transformer model for the calculation of electromagnetic transients is presented. The model consists of a set of state equations solved with the trapezoidal rule of integration in order to obtain an equivalent Norton circuit at the transformer terminals. Thus the transformer model can be easily interfaced with an electromagnetic transients program. Its main features are: (a) the basic elements for the winding model are the turns, (b) the complete model includes the losses due to eddy currents in the windings and in the iron core, (c) the solution of the state equations is obtained in decoupled iterations. For validation, the frequency response of the model is compared with tests on several transformers. Applications to the calculation of transients are given for illustration.

Keywords: Transformer modeling, Electromagnetic transients, Eddy currents, Laminations.

\section{INTRODUCTION}

There exists a wide variety of transformer models, however, no transformer model for the study of electromagnetic transients, adequate for a wider range of frequencies, is yet available. This paper presents a complete transformer model which is suitable for the calculation of transients.

The main streams in the computer modeling for analysis and design of transformers can be classified as:

a) Modeling based on self and mutual inductances. The first analytical attempt following this approach was presented by Rabins ${ }^{11]}$ followed by many others such as Fergestad and Henriksen ${ }^{[2],[3]}$ and continued recently by Wilcox et al. ${ }^{[4]-[6]}$ There are very accurate formulae available for the calculation of self and mutual inductances for the windings, sections, or turns of transformers. However, because of the presence of the iron core, the numerical values of self and mutual inductances are very close and may result in ill-conditioned equations. The problem of illconditioning has been adequately solved in transient simulations by subtracting a common flux in the calculation of self and mutual inductances ${ }^{[3],[7]-[9]}$; see reference [3] for details. The same methodology can be applied in the models of references [4] to [6] In fact, subtracting a large common quantity from the self and mutual inductances is equivalent to the direct use of leakage inductances.

b) Modeling based on leakage inductances. This approach was initiated by Blume ${ }^{[10]}$ and improved by many others; see references [11] to [13]. The three-phase multi-winding generalization was presented by Brandwajn et al. ${ }^{[14]}$ Dugan and others ${ }^{[15]}$ used the same technique for the modeling of multisection transformers. These models represent adequately the leakage inductance of the transformer (i.e. load or short circuit conditions), but the iron core is not properly included.

93 WM 053-9 PWRD A paper recommended and approved by the IEEE Transformers Committee of the IEEE Power Engineering Society for presentation at the IEEE/PES 1993 Winter Meeting, Columbus, OH, January 31 February 5, 1993. Manuscript submitted August 30 1991; made available for printing November 3, 1992. c) Modeling based on the principle of duality. This approach was introduced by Cherry ${ }^{[16]}$ and generalized by Slemon ${ }^{[17]}$. The iron core can be modeled accurately. However, models based only on this approach have the inconvenience that the leakage inductances are not correctly represented (they are directly derived from the leakage flux neglecting the thickness of the windings). This inaccuracy has been corrected (when the magnetic field is assumed axial) by Edelmann [18] and Krähenbiihl et al. ${ }^{[19]}$ Lately, Arturi ${ }^{[20]}$ has employed this approach in the modeling of highly saturated conditions.

d) Modeling based on measurements. There exists a great number of high frequency transformer models derived from measurements; see, for instance, references [21] to [31]. Tests are made for the determination of the model parameters in the frequency domain or time domain. Models obtained from measurements have the drawback that their performance can only be guaranteed for the tested transformers. Although some general trends can be inferred from the tests, according to design, size, manufacturer, etc., accurate predictions for non-tested transformers cannot be assured.

e) Analysis based on electromagnetic fields. Designers of large transformers use electromagnetic field approaches for the calculation of the design parameters. The technique of finite elements is the most accepted numerical solution for field problems. ${ }^{[32]-[35]}$ There are, however, other techniques available; see references [36] and [37]. There is general agreement that three-dimensional field analyses are necessary in the design process. These methods are impractical for the calculation of transients since they would give very expensive simulations.

The purpose of this paper is twofold: (i) to present a complete, three phase transformer model assembled from the theory developed in references [38] to [41], and (ii) to illustrate the performance of the model in the calculation of transients.

The complete model is derived from a combination between the two approaches $b$ and $c$ (leakage inductances and principle of duality) described above. The leakage inductances and the iron core are properly included in the model. Since we want a model appropriate for high frequency transients, the parameters can be calculated on a turn-to-turn basis or using sections with a small number of turns. All capacitances between turns (or sections) on the same leg and capacitances from the turns to ground (core) are included in the model. We have also included in the model the losses produced by the eddy currents in the laminations of the iron core and in the windings (skin and proximity effects). The magnetization of the iron core can be modeled as a nonlinear function between flux and current. The complete model is written as a set of state equations (some of them nonlinear) that are solved iteratively in a decoupled way. The terminal model for the transformer is a Norton equivalent and can thus be easily interfaced with an electromagnetic transients program.

\section{DESCRIPTION OF THE TRANSFORMER MODEL}

Windings
Parameters
The windings parameters (inductances and capacitances) are calculated
starting with the turns as follows (see reference [38] for turn-to-turn and
[41] for sections):
A) Leakage inductances between pairs of turns (or sections) are the
basis of the model for the inductive phenomena in the window of
the transformer. For their calculation we use an image method.
This leads to a loop inductance matrix which describes the voltage
drop in the turns.
drop in the turns. 
B) The inter-turn (or inter-section) capacitances and the capacitances to ground are calculated with the charge simulation method. We get a turn capacitance matrix which can be transformed into a node capacitance matrix.

When the parameters are calculated on a turn-to-turn basis several turns can be lumped in series to form sections. Thus, we can reduce the turnto-turn model to a manageable size as shown in reference [39].

\section{Eddy Current Modeling}

A frequency dependent resistance matrix accounts for the damping produced by the eddy currents in the turns. This matrix is obtained from the total losses in the windings. The matrix is also calculated on a turnto-turn or section basis and, as in the previous cases, it can be reduced by lumping a number of turns or sections in series. A state equation for the voltage drop (due to the eddy currents in the windings) is derived from the frequency variation of the resistance matrix. This permits to include the damping in the complete transformer model; see reference [41].

\section{State Equation Representation}

For each leg in a transformer we have an independent equation describing the voltage in the windings. For leg $a$, the total voltage is given by (see reference [39]):

$$
\mathbf{v}_{a}=\mathbf{w}_{a} e_{a}+\mathbf{v}_{r}^{a}+\mathbf{L}_{a}^{\prime \prime} \frac{d}{d t} \mathbf{i}_{a}
$$

where $\mathbf{v}_{a}$ is the vector containing the total voltages in the sections of leg $a ; \mathbf{w}_{a}$ corresponds to the vector with the number of turns per section; $e_{a}$ represents the common voltage (due to the flux in the limb); $\mathbf{v}_{r}^{a}$ is the vector of the voltage drops due to the eddy currents in the windings; $\mathbf{L}^{\prime \prime} a$ stands for the loop (leakage like) inductance matrix; and, $i_{a}$ is the vector containing the current in the sections.

The differential equation describing the behavior of the voltages and currents in the capacitances (for $\operatorname{leg} a$ ) is:

$$
\mathrm{i}_{C \text { node }}^{a}=\mathbf{C}_{\text {node }}^{a} \frac{d}{d t} \mathbf{v}_{\text {node }}^{a}
$$

where the nodal capacitance matrix $\mathbf{C}_{\text {node }}$ can be obtained from the turn capacitances by shifting half of each capacitance to the two ends of the turn (see reference [39]). We note that the capacitive state equation is in the nodal reference frame while the inductive equation is in the branch reference frame. To combine the two, we use a power-invariant transformation to bring the inductive equation to the common nodal reference frame.

For the voltage drop $v_{r}^{a}$ due to the eddy currents in the windings of leg $a$, we have the following state equations:

$$
\begin{gathered}
\dot{\mathbf{x}}_{a}=\mathbf{A}_{a} \mathbf{x}_{a}+\mathbf{B}_{a} \mathbf{i}_{a} \\
\mathbf{v}_{r}^{a}=\mathbf{C}_{a} \mathbf{x}_{a}+\mathbf{D}_{a} \mathbf{i}_{a}
\end{gathered}
$$

The elements of the state matrices $\mathbf{A}_{a}, \mathbf{B}_{a}, \mathbf{C}_{a}$ and $\mathbf{D}_{a}$ are computed by the time domain approximation of the frequency dependent resistance matrix. We fit a Foster series circuit to the diagonal elements of the matrix and use the poles of these circuits to obtain basis functions for the realization of the off-diagonal elements. The state variables $x_{a}$ are the currents in the inductances of the Foster circuits. The details of the time domain approximation are reported in reference [41]. A good general review of circuit fitting can be found in reference [42].

\section{Iron Core}

For the iron core we represent the magnetization and the damping produced by the eddy currents in the laminations. Hysteresis is not yet included in the model; its expected effect is an increase of damping of the transients and, possibly, some remanent magnetization. The proposed magnetization model is a nonlinear function between current and magnetic flux. This function can be fitted from tests on the iron core material; see, for instance, reference [43]. The penetration of the eddy currents in the iron core is taken into account by a Cauer equivalent circuit fitted to the frequency dependent expression of the lamination impedance. ${ }^{[40]}$ Figure 1 shows the equivalent circuit for the iron core This circuit can be interpreted as an optimized discretization of the lamination for the selected fitting frequencies; see reference [40] and Appendix A for details on the fitting.

Note that the Cauer circuit shown in Figure 1 has the first shun resistance $R_{1}$ removed. This has been found necessary in order to improve the convergence of the decoupled iterative method used for the calculation of transients with our transformer model. In reference [39] we have shown that keeping the magnetizing current small guaranties convergence. Therefore, a circuit having an inductance in series with the input terminals is more suitable for our purpose. Moreover, the methodology for fitting Cauer circuits described in reference [40] applies to the circuit of Figure 1 almost unchanged. In Appendix $A$ we have described the changes and compared the value of the parameters with the two kinds of fitting. They remain almost the same. However, in the iterative solution of the complete transient model, the fact that the Cauer circuits have a series inductance at their input terminals proved to be crucial to assure convergence.

One major advantage of our model for the laminations, over some other models derived from the same basic impedance equation [44],[48], is that it permits the inclusion of the nonlinearities into the inductances of the electric circuit. To be in full agreement with the principle of duality, the iron core model should have the inductive branches placed longitudinally. Otherwise only the terminal behavior under linear conditions can be accurately represented. The state equations describing the behavior of the Cauer model are derived in Appendix B.

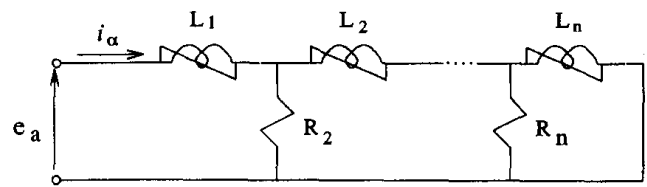

Figure 1. Cauer model for the iron core

\section{Complete Model}

Structure

The complete model for a three-legged transformer is shown schematically in Figure 2. The magnetic model consists of a circuit structure derived from the principle of duality. Each inductor representing a section of the iron core has been substituted by a nonlinear Cauer circuit and the inductors representing the flux path in air have been substituted by a loop inductance matrix. Note that our model does not consider mutual inductances in air between windings wound on different limbs. The magnetic coupling is provided only by the iron core. The connecting points between the iron core circuits and the loop inductance matrices are the points $\alpha$ and $\beta$ which simultaneously represent the leakage flux and the leakage inductance. The application of Kirchhoff's current law (KCL) to nodes 1 and 2 of Figure 2 is very important conceptually. KCLs represent the fact that the sum of currents inside of a window is zero (in the electric equivalent circuit) and correspond to the sum of the mmf's (of the magnetic circuit) around the windows.

The magnetic effects in the air are modeled (as mentioned before) by the loop inductance matrices $\mathbf{L}_{a}, \mathbf{L}_{b}$, and $\mathbf{L}_{c}$ (which do not have an equivalent circuit representation). These matrices account for the leakage inductances between the windings (sections or turns) wound on the same leg. Mathematically, the model has three state equations (one per limb) of the type of equation (1). These three state equations combined with the relations derived from the two KCLs are the central part of the model. All other components interact with them through variables obtained from their solution.

Each nonlinear Cauer circuit in Figure 2 represents the magnetization and eddy current losses in the laminations of a section (leg or yoke) of the iron core. The mathematical model for every Cauer circuit (we have a total of five) is given by the state equations (B-5) of Appendix $B$. Note that the inputs to these circuits are the voltages and the outputs are the currents.

The losses (or damping) due to eddy currents in the windings are represented in Figure 2 by the blocks containing series Foster circuits. The inputs to those blocks are the currents in the windings and the outputs are voltage drops. Mathematically, the model for these losses consists of three sets of state equations of the type of equations (3).

The model for the capacitances between turns (or sections) is directly derived in the nodal reference frame while the inductive model is formulated in the branch reference frame. To interface both models we must convert them to a common reference frame. It is best to convert the inductive equations to the node reference frame by a power-invariant transformation. 


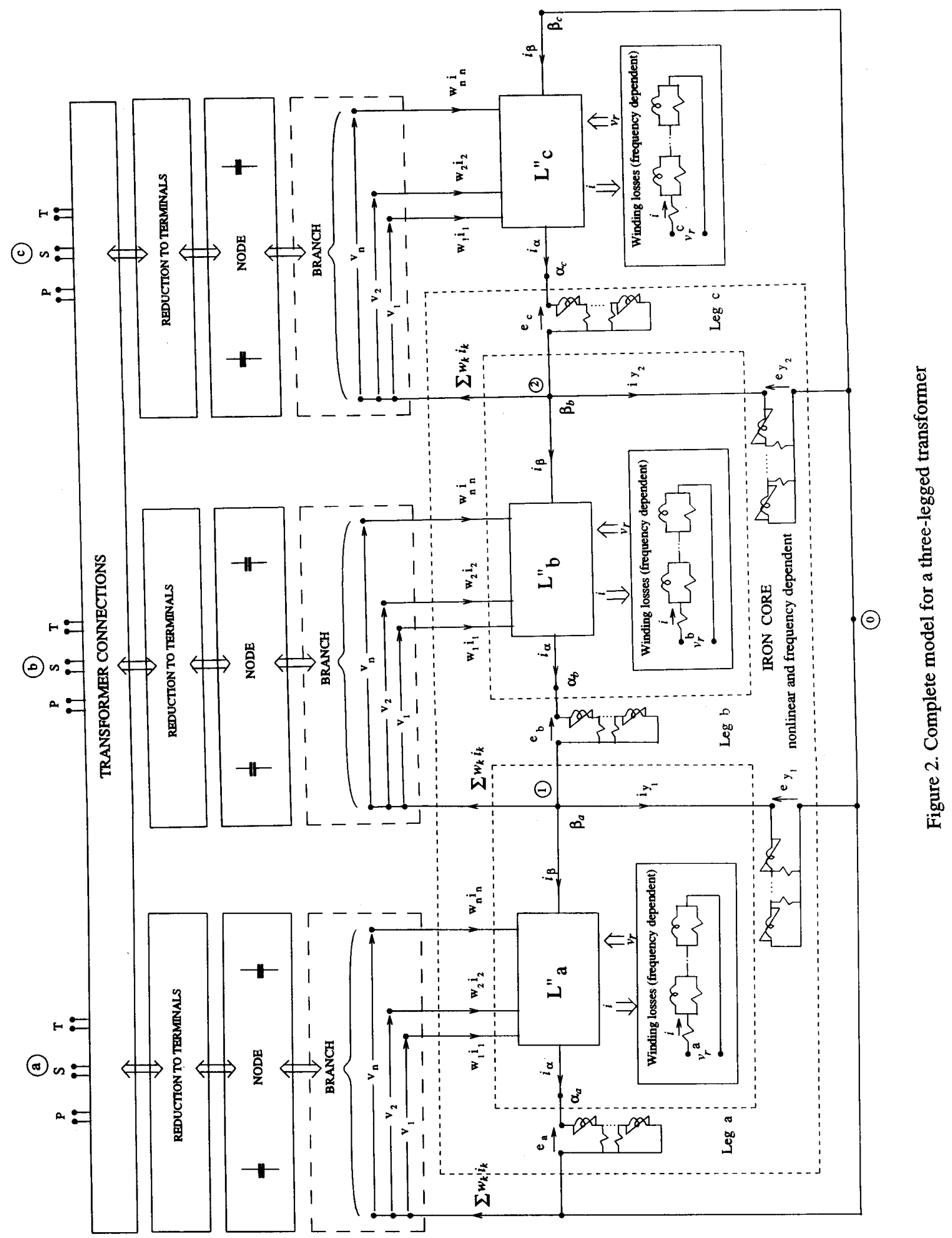


The complete nodal model is conveniently reduced to the external terminals (or nodes) for each leg before the transformer connections ( $\Delta$, $Y$, zig-zag, etc.) are made. The reason is that we can use the same matrices if the three legs are constructed identically. In Figure 2 we have shown a transformer with three legs and three windings but the modeling described in this paper can handle transformers of any number of legs and windings. Moreover, the iron core divisions are not limited to considering the whole leg or yoke as an entity; we can divide it in any number of sections by using more fictitious turns.

\section{Model Solution and Terminal Interfacing}

As the iron core equations are nonlinear, the model requires an iterative solution for every time step. We devised a decoupled iteration process in which we treat independently:

a) the currents and voltages in the turns (or sections) and in the capacitances,

b) the magnetizing currents, and

c) the voltage drops due to eddy currents in the windings.

The flow diagram of the transients program is shown in Figure 3.

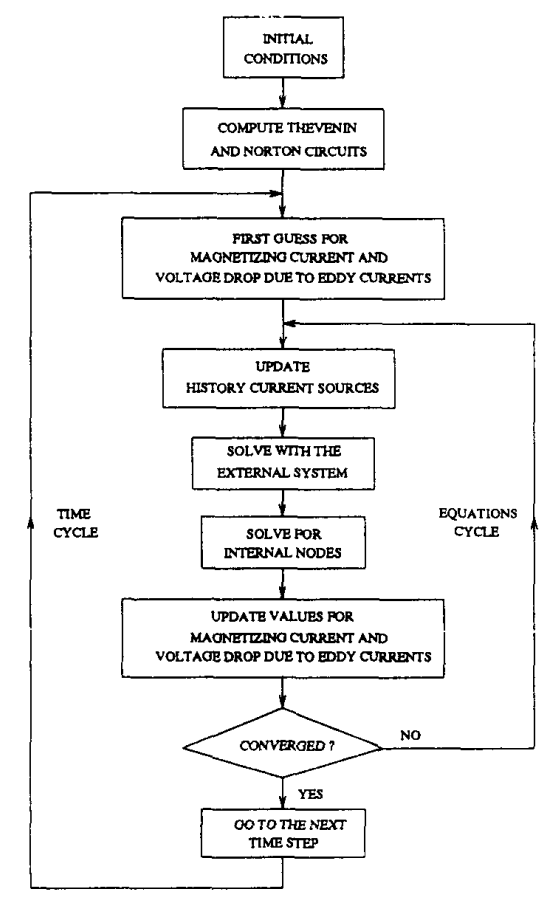

Figure 3. Flow diagram of the transient program

We start by reading the initial conditions for all the state variables: currents in the turns $\left(\mathbf{i}_{a}, \mathbf{i}_{b}, \mathbf{i}_{c}\right)$, voltages in the capacitances $\left(\mathbf{v}_{\text {node }}^{a}, \mathbf{v}_{\text {node }}^{b}\right.$ $\mathbf{v}_{\text {node }}^{c}$ ), currents in the inductances of the Foster circuits $\left(i_{L}^{a}, i_{L}^{b}, i_{L}^{c}\right)$, and currents in the nonlinear inductances representing the iron core sections $\left(\mathbf{i}_{i}^{a}, \mathbf{i}_{i}^{b}, \mathbf{i}_{i}^{c}, \mathbf{i}_{i}^{y_{1}}, \mathbf{i}_{i}^{y_{2}}\right)$.

As the solution of the linear differential equations for the windings is obtained by applying the trapezoidal rule of integration, we obtain Thevenin or Norton equivalent circuits. We write a Thevenin equivalent circuit for the voltage state equation (1) of the windings and Norton equivalent circuits for the rest of the state equations. The details were presented in reference [39]. All conductance matrices remain constant during the whole transient simulation, while the current sources (representing the history) of the elements must be updated at every iteration within a given time step.
The iteration begins by guessing values for the magnetizing currents $\left(i_{\alpha}^{a}, i_{\alpha}^{c}, i_{\alpha}^{c}, i_{y_{1}}, i_{y_{2}}\right)$ and for the voltage drops due to eddy currents in the windings $\left(\mathbf{v}_{r}^{a}, \mathbf{v}_{r}^{b}, \mathbf{v}_{r}^{c}\right)$; see Figure 2. The next step is updating (calculating for the first iteration) the history current sources for all the state variables. Now we can inter-connect the Thevenin and Norton equivalent circuits to form an external Norton circuit reduced to the terminals of the transformer. This circuit can be easily interfaced with an electromagnetic transients program (for example the EMTP) since most of the components are also modeled as Norton equivalent circuits. Subsequently, we calculate the internal voltages and currents in the turns or sections $\left(\mathbf{v}_{a}, \mathbf{v}_{b}, \mathbf{v}_{c}, e_{a}, e_{b}, e_{c}, e_{y_{1}}, e_{y_{2}}, \mathbf{i}_{a}, \mathbf{i}_{b}, \mathbf{i}_{c}, i_{\beta}^{a}, i_{\beta}^{b}, i_{\beta}^{c}\right)$. Note that in this calculation we have included the voltages in the iron core and the currents in the fictitious turns $\beta$.

The iterative process continues with two independent steps:

i) The magnetizing currents are updated using the voltages at the terminals of the Cauer circuits as inputs (see Figure 2). We use a Runge-Kutta method because a Norton equivalent circuit is not needed for the magnetizing part of the model since, in the decoupled solution, only the voltages $\left(e_{a}, e_{b}, e_{c}, e_{y_{1}}, e_{y_{2}}\right)$ are inputs to the nonlinear Cauer circuits.

ii) The voltage drops due to eddy currents in the windings are updated using the currents in the turns $\left(\mathbf{i}_{a}, \mathbf{i}_{b}, \mathbf{i}_{c}\right)$ as inputs. We use the trapezoidal rule of integration since only one (small) matrix is needed and it remains constant during the iterative process.

Next, we get a second approximation for the external Norton equivalent. We repeat the procedure until there is no significant change in the value of the state variables and then we go to the next time step.

\section{Convergence}

In reference [39] we have shown that the iterative cycle converges rapidly when detailed models for the iron core and for the damping due to eddy currents in the windings are not included. Typically we needed 3 to 5 iterations with a tolerance of $10^{-5}$. When the model for the losses due to the eddy currents in the windings was included in the simulation (as in reference [41]), convergence was obtained (for the same tolerance) in 5 to 7 iterations for small time steps and in 8 to 10 for large ones. The number of iterations increased not only because the number of equations increased, but also because the added equations are stiff. Now that a detailed modeling is included for the eddy currents in the iron core, we get convergence in $\mathbf{4}$ to 5 iterations for small time steps and 10 to 11 for large ones. We have also noted that the convergence properties worsened in inverse proportion to saturation. A more detailed discussion on the convergence of the method can be found in Appendix 2 of reference [39] and a still more comprehensive discussion in reference [49].

In addition to the requirement for a small integration time step when we are interested in the simulation of fast transients, there are two limitations in the size of the time step. One is imposed by the decoupled method itself. No convergence is obtained for very large time steps. The other limitation is due to the fact that the Runge-Kutta method is not Astable and thus requires a step size smaller than the smallest time constant. The first limitation can be eliminated with a different (nondecoupled) scheme for the transformer transient model. This may be computationally more expensive, since the size of the Norton equivalent matrix increases significantly. The second limitation can be overcome by using an implicit integration method (for instance, the trapezoidal rule of integration). We have also solved the problem with a two-time-step method, one small time step as required by the transformer model and another (larger one) for the external system; see reference [50].

\section{VALIDATION OF THE TRANSFORMER MODEL}

\section{Parameters}

We have previously validated the parameters (leakage inductances, capacitances and resistances) for the windings independently. The leakage inductances of pairs of turns have been validated against a twodimensional finite elements program in reference [38]. In the same reference the total leakage inductance for a pair of windings was compared against tests on a real transformer and with finite elements as well. For the capacitances we have confirmed that the potential profiles are close to the expected values. The frequency dependent resistance matrix (from which our model is derived) was verified with a well-tested equation in reference [41] and with frequency domain measurements. 


\section{Time Domain Approximations}

For the frequency dependent parameters we have performed time domain approximations obtained from the known frequency responses. Each component of the iron core in the model (Figure 2) can be represented by a Cauer circuit of order 4 if a very accurate response is required up to a frequency of $1 \mathrm{MHz}$ with no saturation; see reference [40] and Appendix A. In the same reference we have shown that in the expressions for the parameters the variables $\omega$ and $\mu$ always appear in the product form $\omega \mu$. Therefore, the frequency response of a Cauer circuit can be assessed as being good for any degree of saturation by simply scaling the frequency axis to keep the product $\omega \mu$ constant. Thus, under saturated conditions the same Cauer circuit is valid for even higher frequencies.

We have shown in reference [41] that a model of the order of 3 times the number of sections in a winding is accurate enough for the representation of the behavior of the winding resistance up to $1 \mathrm{MHz}$.

\section{Frequency Response}

\section{Short Circuit}

The frequency response in short circuit of three transformers was available: a small $2 \mathrm{kVA}$ laboratory transformer, a $75 \mathrm{kVA}$ distribution transformer, and a 93 MVA power transformer. In Figures 4,5 , and 6 we compare the variation with frequency of the computed input impedance of the model against measurements.

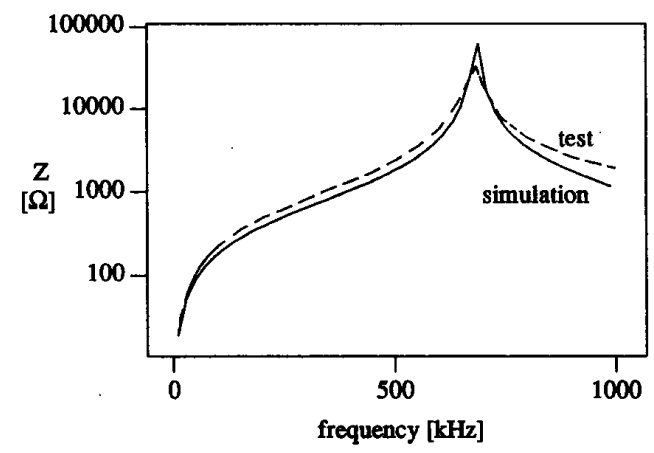

Figure 4. Input impedance for the $2 \mathrm{kVA}$ transformer

From Figure 4 we can see that the overall response of the model for the small transformer (including at resonance) is very satisfactory. For this 2 kVA transformer the complete construction data was available; see Appendix 2 of reference [38]. The parameters of the model were derived on a turn-to-turn basis (as in reference [38]) and reduced to 4 sections per winding (as in reference [39]) for the simulation of Figure 4.

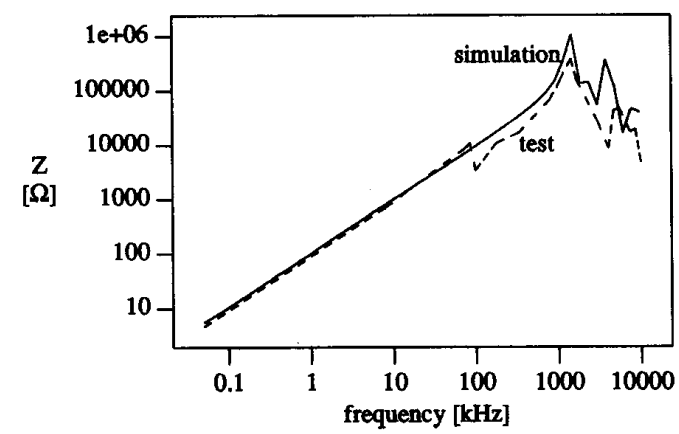

Figure 5. Input impedance for the $75 \mathrm{kVA}$ transformer

In Figure 5 we show the variation of the input impedance for the 75 kVA distribution transformer described in reference [29]. We note that the response of the model is very good at low frequencies (which is mainly governed by the leakage inductance). The two main resonances are also satisfactorily simulated, however, some smaller peaks have not been obtained. For this case the construction data needed for the estimation of the parameters for our model were not available and we have therefore performed an approximate design. A very efficient procedure for parameter calculations was used (as described in Appendix $C$ of reference [41]) which consists in grouping several turns into sections before applying the procedures of reference [38]. Considering that the construction data was obtained by an approximate design and that the model derived in this paper is intended to be used for the simulation of transients (with an inherent time domain averaging), the model response can be considered as adequate.

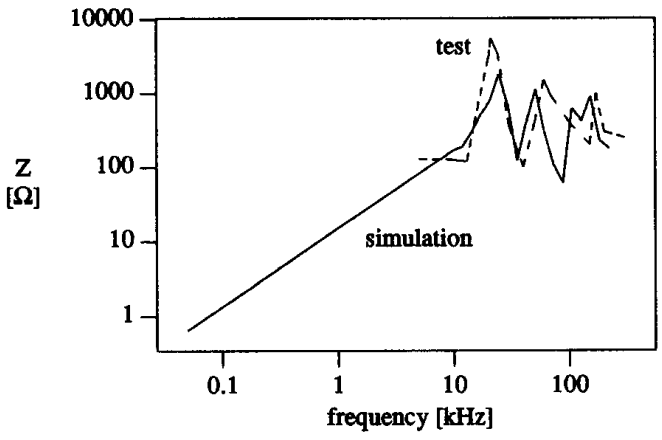

Figure 6. Input impedance for the 93 MVA transformer

The variation of the input impedance of the 93 MVA transformer described in reference [51] is shown in Figure 6 together with the simulation obtained with the model of this paper. We have a reduced frequency range for the tests because the resolution of the measurements has limited the usable points. As in the previous case, the construction data were obtained with an approximate design. Although the model does not show all the resonances at the right locations, the results are satisfactory for the calculation of transients.

Open Circuit

Only the frequency response of the $75 \mathrm{kVA}$ distribution transformer was available in this test case. In Figure 7 we compare the variation of the input impedance with frequency of the model against measurements. While not all resonances are exactly matched, the results are quite satisfactory for a model for time domain simulations.

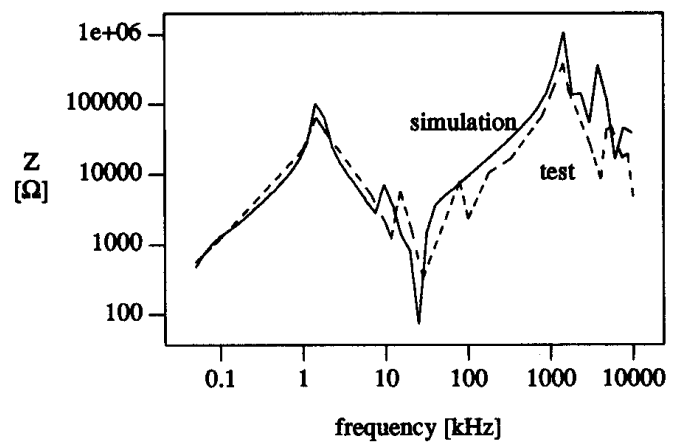

Figure 7. Input impedance for the $75 \mathrm{kVA}$ transformer in open circuit

To assure convergence in the open circuit simulation, we reversed the internal iterations in the model, since before we relied on the fact that the magnetizing current is small compared to the winding current. This condition is normally satisfied, with the exception of the open circuit test at low frequencies or when the transformer is strongly saturated; see Appendix 2 of reference [39] for more details on convergence. For the open circuit simulation at low frequencies, instead of starting the iteration with a small magnetizing current and computing the winding currents later in the iteration, we start with the internal voltage and compute the turn (or section) voltages in the iteration. In the example shown in Figure 7 convergence was obtained in 3 to 9 iterations.

It is interesting to note that the short circuit test results show resonances at significantly higher frequencies than open circuit tests. Reference [29] presents a detailed discussion based on measurements. 
236

\section{APPLICATIONS TO THE CALCULATION OF TRANSIENTS}

Below we present simulations on the small, three-legged, three phase transformer of reference [38], having 118 turns per winding in two layers. We have considered for the simulations two sections per winding with 59 turns each. The model for the eddy currents in the windings is of order 2 (Foster circuits with two inductances). For the five iron core sections we use "incomplete" Cauer circuits, as in Figure 1, of order 6 (six sublaminations). Saturation is considered, as mentioned above, using the nonlinear function described in Appendix B.

Figure 8 shows the transient obtained when energizing the transformer from a balanced sinusoidal voltage source. The transformer connection is $\mathrm{Yg} / \mathrm{Yg}$ with the secondary in open circuit.

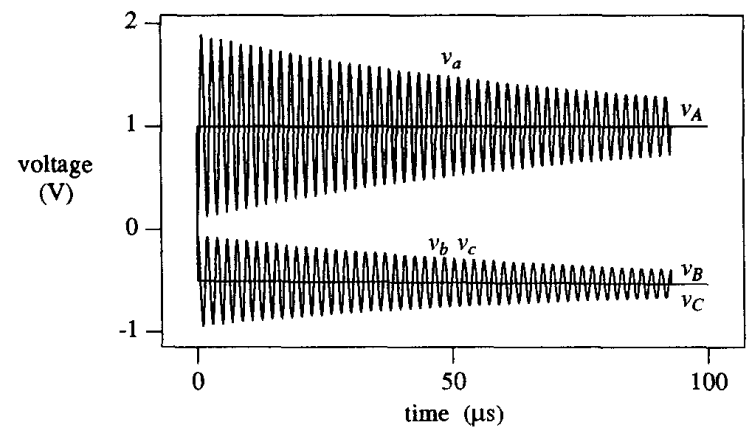

Figure 8. Primary and secondary voltages during energization

In the figure, upper case subscripts have been used for the primary voltages and lower case subscripts for the secondary voltages. The primary and secondary voltages in phases $b$ and $c$ are indistinguishable during the short simulation time when $v_{A}$ and $v_{B}=v_{C}$ remain virtually constant. The frequency of oscillation is of the order of megahertzs and matches the value calculated from $\omega=1 / \sqrt{L_{\text {leak }} C}$ with the known parameters $C=10^{-10} \mathrm{~F}$ and $L_{\text {leak }}=10^{-4} \mathrm{H}$. Table 1 shows the number of iterations (related to the decoupled nature of the computations) required to obtain convergence within a given time step.

\begin{tabular}{|c|c|c|}
\hline$\Delta \mathrm{t}[\mu \mathrm{s}]$ & ITERATIONS & COMMENT \\
\hline 0.1 & $4-5$ & case shown in Figure 5 \\
1 & $5-6$ & \\
10 & $5-7$ & \\
100 & $6-8$ & \\
200 & $7-9$ & Runge-Kutta fails \\
\hline
\end{tabular}

Table 1. Number of iterations for different time steps

As a second example we show in Figure 9 the simulation of a sequential energization followed by current chopping. The transformer was now connected in $\mathrm{Yg} / \Delta$ with the secondary in open circuit. The switching sequence is as follows: phase $a$ is connected at $t=0$ followed by phase $b$ which is connected at $t=0.02 \mathrm{~ms}$; next, phase $c$ is connected at $t=0.04 \mathrm{~ms}$ and, finally, the whole transformer is disconnected at $t=0.06 \mathrm{~ms}$.

We can see from Figure 9 that even when phases $b$ and $c$ are still disconnected from the source there exists a voltage in both primary and secondary. This voltage is induced by the flux in the iron core. The oscillations before disconnection are, as in Figure 8, between leakage inductances and capacitances. After disconnection, the main voltage oscillations are much slower since they are related to the magnetizing inductances. Their frequency is of the order of tens of $\mathrm{kHz}$. This is in accordance with the expected value, obtained with $L_{\text {mag }}=1 \mathrm{H}$. Convergence of the decoupled method was obtained for all integration steps in 4 to 6 iterations.

We have tested the behavior of the transformer model up to several cycles (of power frequency) with different time steps and the results have been fully satisfactory.
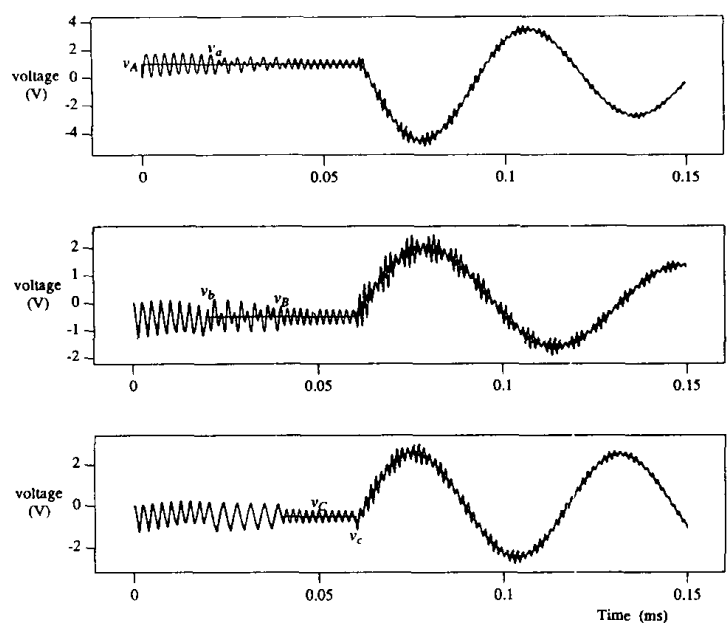

Figure 9. Voltages during sequential energization and current chopping

The next example is the energization of the transformer connected $\mathrm{Yg} / \mathrm{Yg}$, with its secondary in short circuit at $t=0$ and fault clearing at zero current crossing. Inter-turn capacitances were neglected in this simulation. After clearing the fault a $3 \mu \mathrm{F}$ capacitance to ground remains connected to the secondary. In Figure 10 we show the simulated transient recovery voltage (TRV), Figure 10a presents the case when the eddy currents in the windings are included and Figure $10 \mathrm{~b}$ when they are neglected. The frequency of oscillation is governed by the external capacitance (of bus bars, bushings and between the contacts of the circuit breaker) and the transformer leakage inductance. Thus with $L_{\text {leak }}=4.3 \times 10^{-4} \mathrm{H}$ and $C=3 \mu \mathrm{F}$ we get $f \approx 4000 \mathrm{~Hz}$. The integration step was $50 \mu$ s and convergence was obtained in 5 to 6 iterations. From the figure, we can appreciate the significance of including the effect of the eddy currents in the windings. In the case where the effect of the eddy currents is included (case (a) in Figure 10) it appears that two frequencies produce a cancellation effect. Coincidentally, this happens when the source voltage is at its minimum.

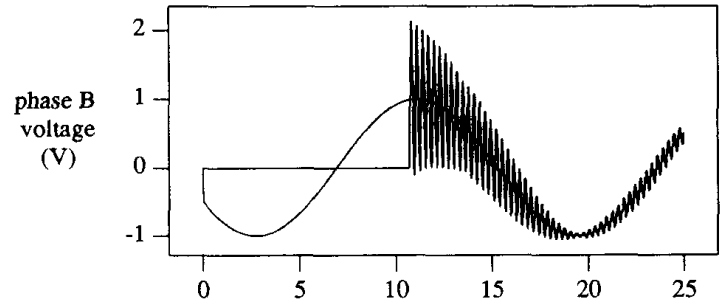

(a)

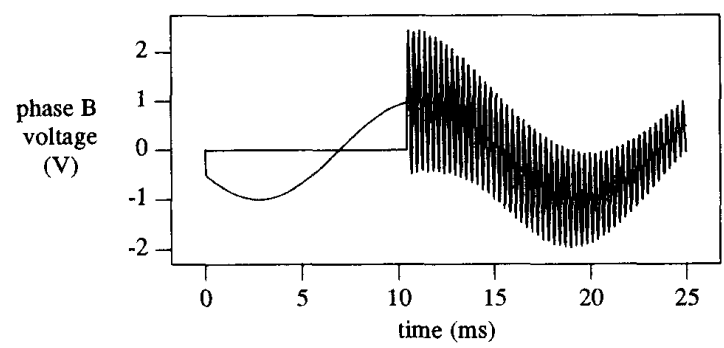

(b)

Figure 10 Transient recovery voltage with a $3 \mu \mathrm{F}$ external capacitance.

(a) Eddy currents in the windings included

(b) Eddy currents in the windings neglected 
Figure 11 shows the simulation of the inrush current obtained when energizing the transformer (from a balanced voltage source of $110 \mathrm{~V}$ ) with its secondary in open circuit. We can see that the current taken by the transformer is several times the magnetizing current. As expected, the transient inrush current is very lightly damped. The results presented here are in agreement with the fact that the inrush current may last for several minutes. In this transient the effect of eddy currents in the windings is not significant. The damping, for the case presented here, is mainly due to the low frequency resistance of the windings. Identical results were obtained when we included the eddy current effects in the windings.

We have used an integration step of $100 \mu \mathrm{s}$. Convergence is obtained in 5 to 6 iterations in the regions where saturation is not important. In the regions where saturation plays an important part, convergence is obtained in an increasing number of iterations reaching a maximum of 85 to 93 at points corresponding to the peak values of the current.

phase B

current

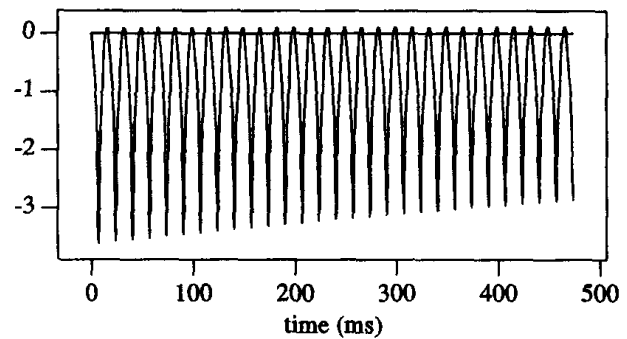

Figure 11 Inrush currents during energization in open circuit

\section{CONCLUSIONS}

A complete, three phase transformer model for the calculation of electromagnetic transients has been presented. The main features of the model described in the paper are:

- the basic elements for the calculation of parameters (inductances and capacitances) are the turns so that internal resonances are fully taken into account,

- the model includes the losses due to eddy currents in the windings,

- saturation and the damping produced by eddy currents in the iron core (laminations) are represented,

- the iterative solution is obtained in a decoupled way for the windings, iron core, and voltage drop due to the currents in the windings,

- voltages and currents at any points in the windings can be displayed.

The model is expressed as a set of state equations solved for the windings with the trapezoidal rule of integration in order to obtain an equivalent Norton circuit at the transformer terminals. Thus, the transformer model can be easily interfaced with an electromagnetic transients program.

For validation, every component of the transformer model has been tested independently against measurements in references [38] to [41]. In this paper the complete and assembled model has been validated with frequency domain measurements. Transient simulations have been performed for illustration and further validation.

\section{ACKNOWLEDGEMENTS}

Financial support by the Natural Sciences and Engineering Research Council of Canada is gratefully acknowledged. The first author wishes to express his gratitude to the Instituto Politécnico Nacional, Mexico, for the study leave at the University of Toronto.

We would like to thank Mr. Arun Narang from the Research Division of Ontario Hydro for his assistance in the measurements of the frequency response and for valuable advice and discussions.

\section{RETERENCES}

[1] L. Rabins, "Transformer Reactance Calculations with Digital Computers", AIEE Transactions, Vol. 75 Pt. I, July 1956, pp. 261-267.

[2] P.I. Fergestad and T. Henriken, "Transient Oscillations in Multiwinding Transformer", IEEE Transactions on Power Apparatus and Systems, Vol. PAS-93, March/April 1974, pp. 500509. P.L. Fergestad and T. Henriksen, Inductances for the Calculation of Transient Oscillation in Transfomers", IEEE Transactions on Power Apparatus and Systems, Vol. PAS-93, March/April 1974, pp. 510-517.

[4] D.J. Wilcox, M. Conlon, and W.G. Hurley, "Calculation of Self and Mutual Impedances for Coils on Femomagnetic Cores", IEE Proceedings, Vol. 135, Pt. A, No. 7, September 1988, pp. 470-476.

[5] D.J. Wilcox, W.G. Hurley, and M. Conlon, "Calculation of Self and Mutual Impodances Between Sections of Transformer Windings", IEE Proceeding, Vol. 136, Pt. C, No. 5, September 1989, Pp. 308-314.

[6] DJ. Wilcox, "Theory of Transformer Modelling Using Modal Analysis", IEE Proceeding, Vol. 138, Pt. C, No. 2, March 1991, pp. 121-128.

[7] W.J. McNutt, T.J. Blalock, and R.A. Hinton, "Response of Transformer Windings to System Voltages", IEEB Transactions on Power Apparatus and Systems, Vol. PAS-93, No. 2, March/April 1974, pp. 457-467.

[8] R.C. Degeneff, "A General Method for Determining Resonances in Transformer Windings", IEEE Transactions on Power Apparatus and Systems, Vol. PAS-96, No. 2, March/April 1977, Pp. 423-430.

[9] R.C. Degeneff, M.R. Gutienrez, and P.J. McKenny, "A Method for Constructing Reduced Order Transformer Models for System Studies from Detailed Lumped Parameter Models", IREE Transactions on Power Delivery, Vol. 7, No. 2, April 1992, pp. 649-655.

[10] L.F. Blume, editor, "Transformer Engineering", Second Edition, John Wiley and Sons, 1951.

[11] J.H. McWhirter, C.D. Fahmkopf, and J.H. Steele, "Determination of Impulse Stresses within Transformer Windings by Computers", AIER Transactions on Power Apparatus and Systems, February 1957, pp. 1267-1274.

[12] R.B. Shipley, D. Colemen, and CF. Watts, "Transformer Circuits for Digital Studies", AIEE Transactions Pt. III (Power Apparatus and Systems), Vol-81, February 1963, pp. 1028-1031.

[13] H. W. Dommel, "Transformer Models in the Simulation of Electromagnetic Transients", Proc. Sth Power Systems Computation Conference, 1975, Sept. 1-5, Paper 3.1/4.

[14] V. Brandwajn, H.W. Dommel, and I.I Dommel, "Matrix Representation of Three-Phase N-Winding Transformers for Steady-State and Transient Studies", IREE Transactions on Power Apparatus and Systems, Vol. PAS-101, No. 6, June 1982, pp. 1369-1378.

[15] R.C. Dugan, R. Gabrik, J.C. Wright, and K.W. Patten, "Validated Techniques for Modeling Shell-Form EHV Transformers", IEEE Transactions on Power Delivery, Vol. PWRD-4, No. 2, April 1989, pp. 1070-1078.

[16] E. Colin Cherry, "The Duality Between Interlinked Electric and Magnetic Circuit and the Formation of Transformer Equivalent Circuits", Proc. of the Physical Society, Vol. (B) 62, Feb. 1949, pp. 101-111.

[17] G.R. Slemon, "Equivalent Circuits for Transformers and Machines Including Nonlinear Effects", Proc. IEE, Part IV, Vol. 100, 1953, pp. 129-143.

[18] H Edelmann. "Anschauliche Emittlung von Transformator-Ersatzschaltbildem", Arch. elektr. Übertragung, Vol. 13, 1959, pp. 253-261.

[19] L. Krähenbühl, B. Kulicke, and A. Webs, "Simulationsmodell eines Mehrwicklungstraneformators zur Untersuchung von Sattigungsvorgangen",

[20] C.M. Arturi, "Transient Simulation and Analysis of a Three-Phase Siep-Up Transformer Following an Out-of-Phase Synchronization", IEEE Transactions on Power Delivery, Vol-6, No. 1, January 1991, pp. 196-207.

[21] M.D'Amore and M. Salemo, "Simplified Models for Simulating Transformer Windings Subject to Impulse Voltage", paper No. A 79 431-8, presented at the IEER PES Summer Meeting July 15-20, 1979.

[22] E.P. Dick and W. Watson, "Transformer Models for Transient Studies Based on Field Measurements", IERE Transactions on Power Apparatus and Systems, Vol. PAS-100, No. 1, January 1981, pp. 409-418.

[23] S. Cristina, M.D'Amore, and M. Salermo, "Digital Simulator of Transformer Windings Subject to Impulse Voltage", IEE Proceedings, Vol. 129, Pt. C, No. 4 July 1982, pp. 172-176.

[24] A. Keyhani, S.M. Miri, and S. Hao, "Parameter Estimation for Power Transformer Models from Time-Domain Data", IEEE Transactions on Power Delivery, Vol. 1 No. 3, July 1986, pp. 1070-1078.

25] P.T.M. Vaessen, "Transformer Model for High Frequencies", IEEE Transaction on Power Delivery, Vol. 3, No. 4, October 1988, pp. 1761-1768.

[26] Q. Su, R.E. James, and D. Sutanto, "A Z-Transform Model of Transformers for the Study of Electromagnetic Transients in Power Systems", IEBE Transactions on Power Systems, Vol. 5, No. 1, February 1990, pp. 27-33.

[27] A. Keyhari, S.W. Chua, and S.A. Sebo, "Maximum Likelihood Estimation of Transformer High Frequency Parameters from Test Data", IEEE Transactions on Transformer High Frequency Parameters from Test Data
Power Delivery, Vol. 6, No. 2, A pril 1991, pp. 858-865.

[28] Y. Liu, S.A. Sebo, R. Caldecott, D.G. Kasten, and S.E. Wright, "Power Transformer Resonance - Measurements and Prediction", paper presented at the IEEE/PES Winter Meeting, February 3-7, 1991, paper No. 91 WM 048-9 PWRD.

[29] A.O. Soysal, "A Method for Wide Frequency Range Modeling of Power Transformers and Rotating Machines", Proc. IEEE Transmission and Distribution Conference, 1991, pp. 560-566.

[30] A. Morched. L. Marti, and J. Ottevangers, "A High Frequency Transformer Model for the EMTP", paper No. 92 SM 359-0 PWRD presented at the 1992 IREE/PES Summer Meeting. 
[31] A.O. Soysal and A. Semlyen, "Practical Transfer Function Estimation and its Application to Wide Frequency Range Representation of Transformers", paper No. 92 SM 358-2 PWRD presented at the 1992 IEEE/PES Summer Meeting.

[32] P. Silvester and M.V.K. Chari, "Finite Element Solution of Saturable Magnetic Field Problems", IEEE Transactions on Power Apparatus and Systems, Voi. PAS 89, No. 7, September/October 1970, pp. 1642-1651.

[33] M.V.K. Chari, J. D'Angelo, M.A. Palmo, and D.K. Sharma, "Application of Three-Dimensional Electromagnetic Analysis Methods to Electrical Machinery and Devices", IEER Transactions on Energy Conversion, Vol. EC-1, No. 2, Jun 1986, pp. 145-157.

[34] N.A. Demerdash, T.W. Nehl, F.A. Fuad, and O.A. Mohammed, Three Dimensional Finite Element Vector Potential Formulation of Magnetic Fields in Electrical Apparatus", IEEE Transactions on Power Apparatus and Systems, Vol. PAS-100, No. 8, August 1981, pp. 4104-4111.

[35] N.A. Demerdash, T.W. Nehl, F.A. Fuad, and O.A. Mohammed, "Experimental Verification and Application of the Three Dimensional Finite Element Magnetic Vector Potential Method in Electrical Apparatus", IREE Transactions on Power Apparatus and Systems, Vol. PAS-100, No. 8, August 1981, pp. 4112-4122.

[36] R.S. Girgis, A.A. Yanrucci, and J.B Templeton, "Performance Parameters of Power Transformers Using 3D Magnetic Field Calculations", IEEE Transactions on Power Apparatus and Systems, Vol. PAS-103, No. 9, September 1984, pp. 2708-2713.

[37] G. Lian, Y. Ruopin, and C. Pizhang, "An Equivalent Magnetization Surface Current Approach of Calculation 3-Dimensional Leakage Fields of Transformer", IREE Transactions on Power Delivery, Vol. PWRD-2, No. 3, July 1987, pp, 817-822

[38] F. de León and A. Semlyen, "Efficient Calculation of Elementary Parameters of Transformers", IEEE Transactions on Power Delivery, Vol. 7, No. 1, January 1992, pp. 376-383.

[39] F. de Leon and A. Semlyen, "Reduced Order Model for Transformer Transients", IEEE Transactions on Power Delivery, Vol. 7, No. 1, January 1992, pp. 361-369.

[40] F. de Leon and A. Semlyen, "Time Domain Modeling of Eddy Current Effects for Transformer Transients", paper No. 92 WM 251-9 PWRD presented at the 1992 IEEE/PES Winter Meeting.

[41] F. de León and A. Semlyen, "Detailed Modeling of Eddy Current Effects for Transformer Transients", paper subrnitted for presentation at the IEEE/PES 1993 Winter Meeting.

[42] L. Weinberg, "Network Analysis and Synthesis", McGraw-Hill, 1962.

[43] A. Semlyen and N. Rajakovic, "Harmonic Domain Modeling of Laminated Iron Core", IEEE Transactions on Power Delivery, Vol. PWRD-4, No. 1, January Core", IEEE Tran

[44] J. Avila-Rosales and F. Alvarado, "Nonlinear Frequency Dependent Transforme Model for Electromagnetic Transient Studies in Power Systems", IEER Transactions on Power Apparatus and Systems, Vol. PAS-101, No. 11, November 1982.

[45] J. Avila-Rosales and A. Semlyen, "Iron Core Modelling for Electrical Transients", IEEE Transactions on Power Apparatus and Systems, Vol. PAS-104, No. 11, November 1985, pp. 3189-3194.

[46] E.J. Tarasiewicz, A.S. Morched, A. Narang, and E.P. Dick, "Frequency Dependen Eddy Current Models for Nonlinear Iron Cores", paper No. 92 WM 177-6 PWRS presented at the 1992 IEEE/PES Winter Meeting.

[47] J. Lammeraner and M. Stafl, "Eddy Currents", The Chemical Rubber Co. Press, Cleveland, 1966.

[48] R. Stoll, "The Analysis of Eddy Currents", Clarendon Press, Oxford, 1974

[49] F. de Lecin, "Trantformer Model for the study of Electromagnetic Transients", $\mathrm{Ph}$.D. Thesis, University of Toronto, 1992.

[50] A. Semlyen and F. de León, "Computation of Electro-Magnetic Transients Using Dual or Multiple Time Steps", paper No. 92 SM 595-9 PWRS presented at the 1992 IEEE/PES Summer Meeting.

[51] A.O. Soysal and S.A. Sebo, "Wide Frequency Range Performance of Electric Machines", Proceedings of the 19th Annual North American Power Symposium, October 1987, pp. 68-78, IEEE Cat. No. CH 2501-5/87.

\section{APPENDICES}

Appendix A - Lumped Parameter Fitting of the Iron Core

The procedure for fitting an "incomplete" Cauer circuit (with $R_{1}$ removed - see Figure 1) is very similar to the one described in reference [40] for the complete Cauer circuit (including $R_{1}$ - as in Figure 7 of reference [40]). The steps to follow are:

a) We first estimate initial values for the $N$ inductances and $N-1(2,3, \ldots, N)$ resistances.

b) The tterative process starts from the highest frequency by computing a better approximation for $L_{i}$

$$
L_{t}=\frac{Z_{t}\left(\omega_{N}\right)-Z_{r}\left(\omega_{N}\right)}{\mathrm{j} \omega_{N}}
$$

where

$Z_{t}=$ known terminal impedance computed with equation (3) of reference [40]

$Z_{\mathrm{r}}=$ impedance seen at the right of inductance $L_{1}$

c) Evaluate $Z^{\prime},\left(\omega_{i}\right)$ as

$$
Z^{\prime},\left(\omega_{i}\right)=Z_{l}\left(\omega_{i}\right)-\mathrm{j} \omega_{l} L_{1} \quad \text { for } i=1,2, \ldots, N-1
$$

d) Use $Z_{l}^{\prime}\left(\omega_{i}\right)$ instead of $Z_{l}\left(\omega_{i}\right)$ in equation (22) of reference [40]. Thus, we can use the procedure in equations (21) to (30) of that reference to compute better approximations of the remaining $N-1$ inductances and $N-1$ resistances.

We have used the method described here to compute "incomplete" Cauer circuits of orders 2 to 6. Convergonce was obtained in a few iterations (typically less than 10) with a tolerance of $10^{-5}$ for all cases. The errors over a range of $1 \mathrm{MHz}$ are similar to those obtained with the complete Caver circuit (i.e. less than $1 \%$ - see reference [40] for details). In Table A-1 we compare the value of the inductances and conductances of the two kinds of fitting for models of order 6 . Note that the parameters in the two circuits are very similar.

\begin{tabular}{|c||c|c||c|c|}
\hline \multicolumn{1}{|c||}{} & \multicolumn{2}{c||}{ COMPLETE CAUER } & \multicolumn{2}{c|}{ INCOMPLETE CAUER } \\
\cline { 2 - 5 } & $\mathrm{G}$ & $\mathrm{G}$ & $\mathrm{G}$ & $\mathrm{L}$ \\
\hline \hline 1 & 8.494322 & $8.9127344 \mathrm{E}-10$ & - & $5.5536264 \mathrm{E}-10$ \\
2 & 39.59985 & $2.4922988 \mathrm{E}-09$ & 43.46111 & $2.8191072 \mathrm{E}-09$ \\
3 & 96.43762 & $5.2383959 \mathrm{E}-09$ & 104.4496 & $5.5004277 \mathrm{E}-09$ \\
4 & 188.4306 & $1.0356599 \mathrm{E}-08$ & 193.9819 & $1.0501804 \mathrm{E}-08$ \\
5 & 374.0791 & $1.9719748 \mathrm{E}-08$ & 375.4775 & $1.9677607 \mathrm{E}-08$ \\
6 & 694.9191 & $4.1570111 \mathrm{E}-08$ & 690.5519 & $4.1214129 \mathrm{E}-08$ \\
\hline
\end{tabular}

Table A-1. Comparison of parameters of Cauer circuits of order 6

Appendix B - State Equations for the Cauer Circuits

Consider a part of a Cauer circuit as shown in Figure B-1.

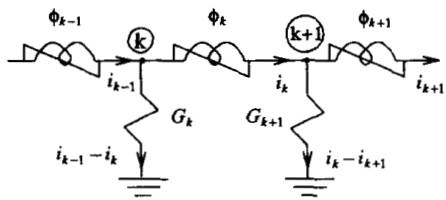

Figure B-1. $k$ th block of the Cauer circuit

The voltage equation for the $k$ th inductance is

$$
\frac{d \phi_{k}}{d t}=v_{k}-v_{k+1}
$$

where $\phi_{k}$ is the flux linkage of inductance $k$. Equation (B-1) can be written as a function of the currents in the inductances as

$$
\frac{d \phi_{k}}{d t}=G_{k}\left(i_{k-1}-i_{k}\right)-G_{k+1}\left(i_{k}-i_{k+1}\right)
$$

The nonlinear characteristics of the material can be expressed as a function of magnetic field intensity $(\mathrm{H})$ and magnetic flux density $(\mathrm{B})$. From test on materials we fit the following function ${ }^{[43\}}$.

$$
H=K_{1} B+K_{17} B^{17}+K_{21} B^{21}
$$

where

$$
\begin{aligned}
& K_{1}=318.32=1 / \mu \\
& K_{17}=0.2181 \\
& K_{21}=0.1353
\end{aligned}
$$

Equation (B-3) can be transformed to a function of current and flux

$$
i=f(\phi)=K_{1} \phi+K_{17}^{\prime} \phi^{17}+K_{21} \phi^{21}
$$

Substituting (B-4) into (B-2) we obtain the desired state equation

$$
\frac{d \phi_{k}}{d t}=G_{k}\left[f\left(\phi_{k-1}\right)-f\left(\phi_{k}\right)\right]-G_{k+1}\left[f\left(\phi_{k}\right)-f\left(\phi_{k+1}\right)\right]
$$

When $k=1$ the left hand side voltage is the forcing function $e_{a}$ (see Figure 1). Then, the first state equation is

$$
\frac{d \phi_{1}}{d t}=e_{a}-G_{2}\left[f\left(\phi_{1}\right)-f\left(\phi_{2}\right)\right]
$$

For the last element, $k=n$, so that

$$
\frac{d \phi_{n}}{d t}=G_{n}\left[f\left(\phi_{n-1}\right)-f\left(\phi_{n}\right)\right]
$$

The state equations (B-5) can be integrated with the Runge-Kutta method of order 4. This method was selected because the forcing function of the Caver circuits is always the voltage and there is no need to obtain for them a Norton equivalent. This, however, imposes a limitation on the step size since the standard Runge-Katta algorithm of order 4 is not A-stable.

Francisco de Leon was bom in Mexico, in 1959. He received his B.Sc. degree and his M.Sc. degree (summa cum laude) from Instituto Politecnico Nacional, Mexico, in 1983 and 1986, respectively. From 1984 to 1987 he worked as a lecturer at the same institute. He obtained a Ph.D degree in 1992, on a topic of transformer modeling, at the University of Toronto. He continued his research there as a postdoctoral fellow until his retum to the I.P.N., Mexico, in September 1992. His main research interests include electromagnetic fields and transients.

Adsm Semlyen ( $F$ '88) was born and educated in Rumania where be obtained a Dipl. Ing. degree and his $\mathrm{Ph} . \mathrm{D}$. He started his career with an electric power utility and held an academic position at the Polytechnic Institute of Timisoara, Rumania. In $1969 \mathrm{~h}$ joined the University of Toronto where he is a professor in the Department of Electrica Engineering. His research interests include the steady state and dynamic analysis of power systems, electromagnetic transients, and power system optimization. 
Discussion

J. R. Martí (University of British Columbia, Canada): The authors are to be congratulated for putting together a practical three-phase transformer model that can simulate a wide range of transient phenomena, including the effects of core saturation and frequency dependence of the iron and copper losses.

One of the components of the model is the windings' series impedance $Z$. The realizability of the diagonal elements of this matrix is assured by synthesizing these elements with Foster's RL series networks. It is clear from the residues condition in network synthesis theory that, as indicated in the paper, the poles of the offdiagonal elements $z_{i j}$ in the $Z$ matrix must be a subset of the set formed by all the poles of all diagonal elements $z_{i i}$. This is a necessary condition for $\mathrm{Z}$ to be a positive real (p.r.) matrix. The sufficient conditions to assure that the full $\mathrm{Z}$ matrix is a p.r. matrix are, however, more complicated. Failure to comply with the p.r. condition on the full $\mathrm{Z}$ matrix could result in numerical instabilities in the transient simulation. The authors comments on this issue would be very much appreciated.

Manuscript received March 1, 1993.

Bruce A. Mork (Michigan Technological University, Houghton, MI), Don L. Stuehm (North Dakota State University, Fargo, ND); This paper presents a very interesting and useful three phase transformer model which should be valid for a wide range of frequencies. The questions asked here are in regard to low-frequency model validation. Inrush simulations provide an excellent time-domain measure of model performance at lower frequencies. Inrush currents depend on winding connections and core configurations and are typically different in each phase of the transformer. Fig. 11 shows the inrush current for phase B. Could the authors provide the inrush current waveforms for phase $A$ and phase $C$ ? Were the windings connected in delta or wye? How did simulated waveforms compare to measured waveforms? Is the model capable of simulating residual fluxes in each of the core limbs? At frequencies up to several $\mathbf{k H z}$, magnetic saturation is prevalent and transformer responses are nonlinear. Referring to Fig. 7, could the authors briefly comment on the use of frequency response as a measure of the model's open-circuit performance in this frequency range?

Manuscript received March 3, 1993.

Francisco de León (Instituto Politecnico Nacional, México) and Adam Semlyen (University of Toronto): We wish to thank the discussers for their appreciative remarks about our paper. The following are clarifications on the questions raised.

Regarding the positive real requirement for the matrix $Z$, we note that the imaginary part $X$ of $Z$ is obtained first through the calculation of the inductance matrix $L$ of the transformer and it is simply augmented by the real part, the matrix $R$, to reflect the winding losses. The way it is calculated, the matrix $R$ is positive definite since it results (in addition to terms representing the d.c. losses and skin effect in the conductors) from the summation of terms that represent the proximity effect due to all other conductors: these are the eddy current losses, proportional to $|H|^{2}$ (and therefore definitely positive), where $H$ is a linear function of all $I$. Since the matrix $R$ is ab ovo positive definite, so is its accurately fitted version, and consequently no instabilities will result in numerical simulations. For details regarding this problem, we refer to reference [41] (equations (7a) and (6)), presented at 1993 IEEE/PES Summer (rather than Winter) Power Meeting.

Regarding the simulation of inrush currents, we agree that these tests serve well for the low frequency validation of the transformer model. We note, however, that the present model, while fairly complete in many respects, does not yet include the representation of hysteresis (intended to be added soon). Therefore, after deenergization, no residual fluxes will remain in a simulation and initial flux values for each leg may have to be set for a new simulation. This, of course, would probably be needed even if hysteresis was modeled. Certainly, its absence in the modeling renders the simulation less accurate and comparisons with measurements confirm only the phenomena related to the existence of large peaks of magnetizing currents due to high levels of saturation.

As correctly pointed out by the discussers, the inrush currents are strongly influenced by many factors, such as transformer connections and core configuration. In the test illustrated in Figure 11, the primary was connected in grounded $Y$. Thus, essentially, the three windings were electrically independent. Figure. A shows the inrush currents in all three phases. Phase $A$ has almost no saturation. The inrush currents in phases $B$ and $C$ show the effect of saturation consistent with the instant of energization. Because of the magnetic coupling of the three legs of the iron core, the magnetizing currents show the effects of the other phases as well. This appears clearly in Figure B which contains the first two cycles of Figure A. For example, the saturation in phase $C$ at around $10 \mathrm{~ms}$ has an effect on the currents in phases $A$ and $B$.

The discussers' remark about the significance of saturation in the lower frequency range is well taken. Tests over a wide frequency range, such as shown in Figure 7, are usually performed at reduced voltages and do therefore not reflect the effect of saturation. Accordingly, simulation results are also shown for unsaturated conditions.

We use this opportunity to indicate two small corrections for the paper: In Table 1 the reference should be to Figure 8 instead of Figure 5 . In the paragraph above Figure 1, the second reference should be [46] rather than [48].

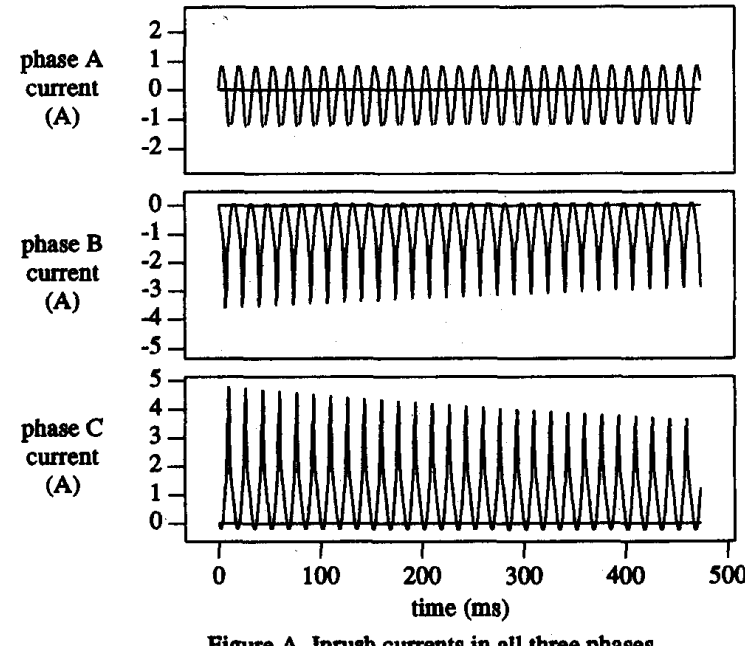

Figure A. Inrush currents in all three phases corresponding to the energization shown in Figure 11

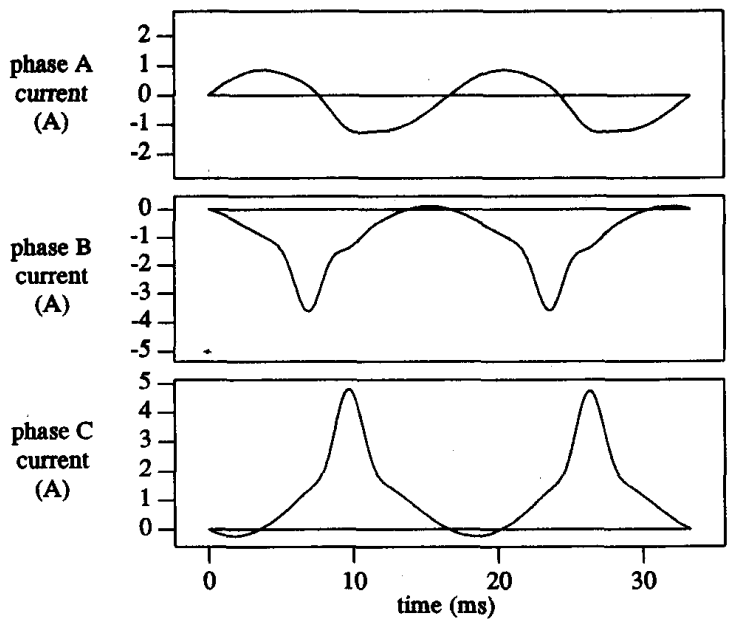

Figure B. First two cycles of Figure A

Manuscript received April 14, 1993. 